\title{
Составление аналитического выражения физического процесса по экспериментальной кривой с изломами
}

\author{
(C) В.Н. Давыдов, С.В. Харитонов, Н.Э. Лугина, К.П. Мельник \\ Томский государственный университет систем управления и радиоэлектроники, \\ 634050 Томск, Россия \\ E-mail: dvn@fet.tusur.ru
}

(Получена 16 января 2017 г. Принята к печати 7 февраля 2017 г.)

\begin{abstract}
Предложена методика составления аналитического выражения экспериментальной кривой, имеющей один или несколько изломов. В зависимости от схемы участия парциальных процессов в результирующем процессе при составлении используются вычисления суммы процессов или их среднего геометрического значения. Для повышения точности аппроксимации в данные выражения введены „функции участия“ процессов, ход которых определяется координатой точки пересечения процессов и „параметром точности“. Их подбором точность аппроксимации устанавливается соответствующей точности измерения экспериментальной кривой. Показано применение предлагаемой методики в аналитических расчетах с комбинацией нескольких процессов, а также при составлении аналитического выражения экспериментальной кривой с изломами. Получаемое таким способом аппроксимирующее выражение характеризуется наглядностью, высокой точностью аппроксимации, физической простотой и может быть использовано для вычисления других свойств полупроводникового прибора.
\end{abstract}

DOI: $10.21883 /$ FTP.2017.09.44886.8402

\section{1. Введение}

При проведении научных исследований часто возникает необходимость аналитического представления экспериментально полученной зависимости, на которой имеются изломы: участки пересечения кривых с разными углами наклона и функциональными зависимостями измеренного параметра от независимой переменной. Особенно часто такая ситуация возникает при исследовании электрических свойств полупроводникового прибора, в частности измерении его вольт-амперной характеристики (BAX) [1-7], когда исследуемая характеристика имеет изломы вверх, вниз или то и другое одновременно. Необходимость получения аналитических выражений таких зависимостей диктуется, с одной стороны, стремлением корректно описать физическую картину исследуемого свойства, а с другой - возможностью с помощью имеющихся экспериментальных данных аналитически предсказать другие свойства полупроводникового прибора. В частности, по известной ВАХ можно описать фотоэлектрические, а после вычисления автокорреляционной функции процесса токопереноса и флуктуационные свойства прибора $[8,9]$. Другая ситуация, в которой требуется детальный анализ экспериментальной зависимости с изломами, - это выявление физических механизмов, проявляющихся в изменении хода кривой в области ее излома. Ее наглядным примером может служить рассмотрение температурной зависимости физической величины, когда имеет место смена доминирующего процесса и связанное с этим изменение энергии активации физического параметра [10-13]. Возможны также изломы на кривых релаксации исследуемого параметра во времени [14], из которых определяют механизмы релаксации и значения их характеристических параметров.
В этой связи перед исследователями встает задача составления аналитического выражения, описывающего экспериментальную кривую во всем исследованном диапазоне независимой переменной, включая области с изломами и изменением наклона. Существуют методы составления аналитического представления экспериментальной зависимости, например, в виде степенного $[15,16]$ или экспоненциального полинома [17], сплайнфункций и т.д. Одним из наиболее используемых методов аппроксимации экспериментальной кривой является ее описание полиномом $n$-й степени с вычислением коэффициентов полинома методом наименьших квадратов. Опыт показывает [15], что даже сравнительно гладкие экспериментальные зависимости аппроксимируются полиномами высокой степени (до $n=10$ и более). Использовать в аналитических расчетах полиномы такой степени неудобно из-за их громоздкости и невозможности предвидеть физический результат аппроксимации ввиду оторванности структуры полинома от узнаваемых математических образов, участвующих в рассматриваемом свойстве физических процессов. К тому же при такой аппроксимации без вычислительной техники не обойтись. Напомним, что ставится задача получения аналитического выражения экспериментальной зависимости, которое можно было бы использовать для вычисления других свойств объекта. Ясно, что эти вычисления предпочтительно вести, понимая структуру формирования вычисляемого свойства. Использование аппроксимации всей кривой полиномом или другой произвольной функцией фактически исключает перечисленное выше.

При составлении аналитической зависимости также обнаруживается, что используемые для описания совместного действия нескольких физических процессов алгебраические операции сложения [18-21], вычисление 
среднего геометрического процесса [22-24] могут дать результирующую кривую, отличающуюся вблизи изломов от экспериментальной кривой до 100\%.

В данной работе предложена методика составления аналитического выражения экспериментальной кривой, имеющей изломы, с использованием операций вычисления среднего геометрического значения или суммарной величины в зависимости от вида экспериментальной кривой и вводимых функций участия процессов, которые позволяют повысить точность аппроксимации в местах изломов кривой.

\section{2. Основные положения методики}

Для пояснения сути предлагаемого метода построения аналитического выражения экспериментальной кривой с изломом рассмотрим случай ломаной кривой, полученной пересечением двух кривых $y_{1}(x)$ и $y_{2}(x)$ в точке $F$ с координатой $x=x_{0}$ (рис. 1). Возможна и обратная задача, когда по известной функциональной зависимости, описывающей физический процесс в реальном объекте, требуется найти аналитическую зависимость величин, формирующих этот процесс, а также определить принцип их объединения. Как следует из рис. 1, объединение двух кривых (процессов) может проявить себя двояко: либо в виде зависимости AFD (обозначим ее $Y_{1}(x)$ ), либо CFB (обозначим как $Y_{2}(x)$ ).

\section{1. Параллельные процессы}

Ломаная кривая AFD, состоящая из двух зависимостей, характеризуется тем, что во всем диапазоне независимой переменной значения функции на этой кривой максимальны. Среди алгебраических операций по объединению двух зависимостей только операция сложения дает аналогичный результат. Поэтому теоретически результирующий процесс в виде верхней кривой AFD можно рассматривать как сумму парциальных процессов [18-20]:

$$
Y_{1}^{(t)}(x)=y_{1}(x)+y_{2}(x) .
$$

С физической точки зрения подобная ситуация имеет место, например, при анализе электрических цепей, когда два процесса (два тока) текут по разным участкам электрической цепи с разными значениями их проводимостей и впоследствии объединяются на малом сопротивлении нагрузки, на котором регистрируется результирующий процесс. Математической операцией по объединению двух параллельно текущих потоков является их алгебраическая сумма, а объединяемые процессы называют параллельными, т. е. текущими независимо друг от друга. Исходя из этого, рассматривая текущие независимые процессы, будем называть их параллельными.

Для многих задач результат вычисления по выражению (1) является правильным. Однако если требуется

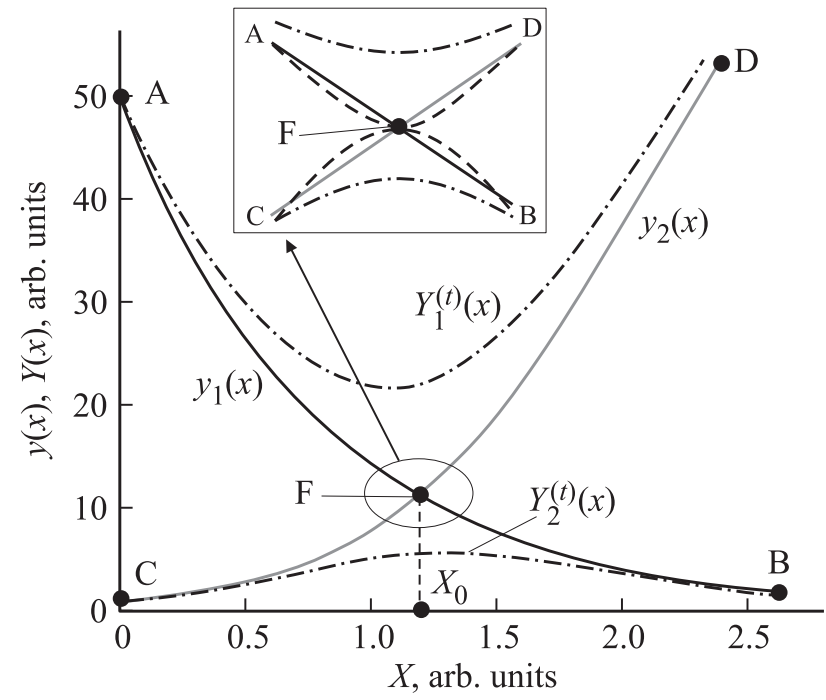

Рис. 1. Графическое представление двух взаимодействующих процессов. На вставке - пунктирными линиями показаны сглаженные зависимости результирующих процессов AFD и $\mathrm{CFB}$, а штрихпунктирными линиями расчетные зависимости $Y_{1}^{(t)}(x)$ и $Y_{2}^{(t)}(x)$.

построить аналитическое выражение для суммарного процесса AFD в виде гладкой кривой, которое давало бы точное значение суммы двух процессов, то использование выражения (1) невозможно. Действительно, как видно из рис. 1, выражение (1) хорошо описывает результирующую кривую AFD в области больших и малых значений аргумента. Однако вблизи точки $x=x_{0}$ значение результирующей функции по (1) в 2 раза больше, чем это следует из графика, и составляет $2 y_{1}\left(x_{0}\right)$. Отметим также, что экспериментально наблюдаемые зависимости со сменой физического процесса вблизи точки излома обычно имеют вид сглаженных кривых. На вставке в увеличенном виде показан ход кривых $y_{1}(x)$, $y_{2}(x)$, а также сглаженные кривые результирующих процессов AFD и CFB вблизи точки пересечения.

Исправить указанные несовпадения расчетных и экспериментально наблюдаемых зависимостей можно введением в (1) функций $f_{1}\left(x, x_{0}\right)$ и $f_{2}\left(x, x_{0}\right)$, описывающих участие процессов $y_{1}(x)$ и $y_{2}(x)$ в формировании результирующего процесса $Y_{1}(x)$ вблизи точки пересечения. Тогда вместо (1) результирующий процесс можно описать выражением

$$
Y_{1}(x)=f_{1}\left(x, x_{0}\right) y_{1}(x)+f_{2}\left(x, x_{0}\right) y_{2}(x) .
$$

По определению, функции участия $f_{1}\left(x, x_{0}\right)$ и $f_{2}\left(x, x_{0}\right)$ должны обладать следующими свойствами: $f_{1}\left(x, x_{0}\right)=\left\{\begin{array}{ll}1, & x<x_{0}, \\ 0, & x>x_{0},\end{array}\right.$ функция $f_{2}\left(x, x_{0}\right)$ ведет себя обратным образом: $f_{2}\left(x, x_{0}\right)=\left\{\begin{array}{ll}0, & x<x_{0}, \\ 1, & x>x_{0} .\end{array}\right.$ В точке $x=x_{0}$ функции равны: $f_{1}\left(x_{0}, x_{0}\right)=f_{2}\left(x_{0}, x_{0}\right)=1 / 2$. 
Перечисленными свойствами обладают функции вида

$$
\begin{gathered}
f_{1}\left(x, x_{0}\right)=\frac{1}{1+\exp \left(\frac{x-x_{0}}{\sigma}\right)}, \\
f_{2}\left(x, x_{0}\right)=1-f_{1}\left(x, x_{0}\right)=\frac{1}{1+\exp \left(\frac{x_{0}-x}{\sigma}\right)} .
\end{gathered}
$$

Параметр $\sigma$ определяет область независимой переменной, в пределах которой значения функций изменяются от нуля до единицы. Чем больше $\sigma$, тем шире диапазон значений аргумента, требуемый для включения в результирующий процесс одного процесса и выключения другого.

Причина повышения точности аппроксимации экспериментальной кривой по предлагаемой методике по сравнению с вычислением алгебраической суммы заключается в том, что вводимые функции участия $f_{1}\left(x, x_{0}\right)$ и $f_{2}\left(x, x_{0}\right)$ „сшивают“ объединяемые процессы $y_{1}(x)$ и $y_{2}(x)$ в пределах диапазона независимой переменной $\sigma$, который выбирается из условия совпадения расчетной кривой с экспериментальной. При объединении же процессов без функций участия диапазон перехода одного процесса в другой $\left(x_{1} ; x_{2}\right)$ задается условиями: $y_{1}\left(x_{1}\right) \gg y_{2}\left(x_{1}\right)$ или $y_{2}\left(x_{2}\right) \gg y_{1}\left(x_{2}\right)$ и потому оказывается значительно больше $\sigma$. Чем меньше наклоны процессов $y_{1}(x)$ и $y_{2}(x)$ к оси абсцисс, тем больше диапазон сшивания процессов и больше ошибка аппроксимации экспериментальной зависимости AFD по формуле алгебраического суммирования без функций участия.

\section{2. Последовательные процессы}

2.2.1. Пространственно-совмещенные процессы. Для ломаной кривой CFB - $Y_{2}(x)-$ характерно, что значения функции на ней во всем диапазоне независимой переменной меньше значений объединяемых функций $y_{1}(x)$ и $y_{2}(x)$. Среди простых алгебраических операций по объединению двух переменных величин только операция вычисления среднего геометрического значения дает такой же результат. Поэтому для нижней кривой результирующий процесс необходимо рассматривать как обратную величину суммы обратных парциальных процессов. К такому же выводу приводит рассмотрение аналогии в виде протекания тока по цепи, составленной из двух последовательно соединенных проводимостей разной величины. В этом случае результирующий ток может быть найден как среднее геометрическое двух токов, каждый из которых определяется одной из заданных проводимостей. Поэтому результирующий процесс необходимо рассматривать как обратный сумме обратных парциальных процессов:

$$
Y_{2}^{(t)}(x)=\frac{y_{1}(x) y_{2}(x)}{y_{1}(x)+y_{2}(x)} .
$$

Физически этот результат означает, что при изменении независимой переменной один парциальный процесс переходит во второй. По этой причине их можно назвать последовательными.
Выражение (4) хорошо описывает экспериментальную кривую CFB в области малых и больших значений аргумента, но вблизи точки $x=x_{0}$ (4) дает значение меньшее, чем это следует из рис. $1: y_{1}\left(x_{0}\right) / 2$. Как и в случае параллельных процессов, это несовпадение также можно исключить введением в (4) функций $f_{1}\left(x, x_{0}\right)$ и $f_{2}\left(x, x_{0}\right)$, описывающих участие процессов $y_{1}(x)$ и $y_{2}(x)$ в результирующем процессе вблизи точки пересечения. Тогда аналитическое выражение для математического описания результирующего физического процесса, состоящего из двух последовательных процессов, примет вид

$$
Y_{2}(x)=\frac{y_{1}(x) y_{2}(x)}{f_{1}\left(x, x_{0}\right) y_{1}(x)+\left[1-f_{1}\left(x, x_{0}\right)\right] y_{2}(x)} .
$$

Можно показать, что в этом случае вблизи точки пересечения каждый из участвующих процессов изменяется и описывается выражениями

$$
y_{1}(x) \rightarrow \frac{y_{1}(x)}{\left[1-f_{1}\left(x, x_{0}\right)\right]}, \quad y_{2}(x) \rightarrow \frac{y_{2}(x)}{f_{1}\left(x, x_{0}\right)} .
$$

\subsection{2. Пространственно-разнесенные процессы.} Если рассматриваемые процессы формирования исследуемого свойства пространственно разнесены, то задача составления аналитического выражения для последовательных процессов может быть решена аналитически без привлечения функций участия. Такая ситуация характерна, например, при описании BAX двухслойных структур типа „металл-диэлектрикполупроводник“ на постоянном токе (рис. 2), в которых диэлектрик обладает утечкой [8].

Действительно, как следует из рис. 2, при последовательном соединении двух активных сопротивлений с различными $\mathrm{BAX}: j_{1}\left(V_{1}\right)$ и $j_{2}\left(V_{2}\right)$ полный ток в цепи находится по выражению

$$
\begin{aligned}
j(V) & =\frac{V}{R_{1}+R_{2}}=\frac{1}{\frac{R_{1}}{V_{1}}\left(\frac{V_{1}}{V}\right)+\frac{R_{2}}{V_{2}}\left(\frac{V_{2}}{V}\right)} \\
& =\frac{1}{\frac{1}{j_{1}\left(V_{1}\right)}\left(\frac{V_{1}}{V}\right)+\frac{1}{j_{2}\left(V_{2}\right)}\left(\frac{V_{2}}{V}\right)} .
\end{aligned}
$$

Здесь $V_{1}+V_{2}=V$ - падения напряжений от источника постоянного напряжения $V$ на первом $V_{1}$ и втором $V_{2}$

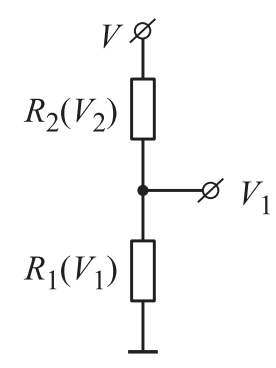

Рис. 2. Эквивалентная схема МДП-структуры с утечкой на постоянном токе. 
элементах соответственно. Отсюда найдем выражение для ВАХ цепочки из двух пространственно-разделенных элементов, соединенных последовательно:

$$
j(V)=\frac{j_{1}\left(V_{1}\right) j_{2}\left(V_{2}\right)}{j_{1}\left(V_{1}\right)\left(1-\frac{V_{1}}{V}\right)+j_{2}\left(V_{2}\right)\left(\frac{V_{1}}{V}\right)} .
$$

Нетрудно видеть, что конструкция выражения (6) аналогична выражению (5), полученному для пространственно неразделяемых процессов, с той лишь особенностью, что для получения (6) в окончательном виде его необходимо дополнить выражением для определения падения напряжения на одном из рассматриваемых элементов. Это можно сделать, если известны механизмы формирования электрического поля в каждом из рассматриваемых элементов $[8,15]$.

\section{3. Применение методики В экспериментальных исследованиях}

Как следует из приведенного выше описания, предлагаемый метод свободен от недостатков аппроксимации всей кривой, поскольку в его основе лежит положение о том, что излом экспериментальной кривой означает смену механизма, формирующего исследуемое физическое свойство. Поэтому вместо аппроксимации всей кривой целесообразно строить аппроксимационные зависимости ее частей (отдельных физических процессов), на которые эта кривая разбивается изломами. По числу изломов можно оценить количество процессов, участвующих в формировании исследуемого свойства. На каждом участке аппроксимационная зависимость описывается простыми, „узнаваемыми“ функциями. Аппроксимационную же зависимость всей кривой получают сшиванием этих функций функциями участия с использованием признаков параллельности или последовательности объединяемых процессов по выражениям (2) или (5). Получаемое таким способом аппроксиманционое выражение всей кривой приобретает структуру, аналогичную структуре экспериментальной кривой, физически прозрачную и удобную для дальнейших математических преобразований $[8,9,15]$.

Продемонстрируем методику аппроксимации экспериментальной зависимости на конкретном примере. На рис. 3 (кривая 1) представлена дифференциальная $\mathrm{BAX}$ поликристалла $n$-CdSe, измеренная в диапазоне напряжения $0<V<10 \mathrm{~B}$ на частоте 35 Гц тестового сигнала амплитудой $1 \mathrm{mB}$. Измерения выполнены с помощью аппаратно-программного комплекca [25]. Как следует из рисунка, полученную зависимость можно рассматривать как результат объединения трех кривых. В диапазоне напряжений $V<V_{1}=3.65$ В BAX хорошо описывается экспоненциальной зависимостью $I_{1}(V)=k_{1} \exp (\alpha V)=0.50 \exp (V / 1.30)$, в диапазоне $V_{1}<V<V_{2}=6.00$ В экспериментальная характеристика аппроксимируется прямой вида $I_{2}(V)=I_{2}^{0}+k_{2} V$ $=5.80+0.75 \cdot V$, а в области значений напряжений

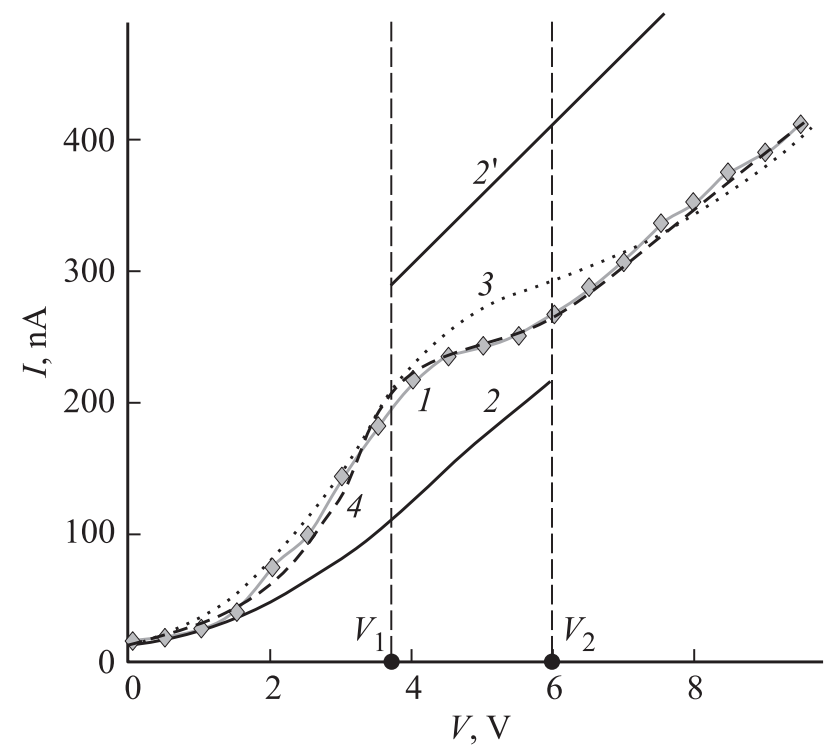

Рис. 3. Зависимость тока через образец $n$-CdSe от напряжения смещения $V$ на частоте 35 Гц, а также ее математическая интерполяция без использования функции участия и с ней.

$V>V_{2}$ ВАХ имеет вид прямой линии: $I_{3}(V)=1.70 \cdot V$. Таким образом, в эксперименте объединены в единый три процесса, первые два из которых взаимодействуют по последовательной схеме, а второй и третий - по параллельной. Тогда, согласно (2) и (5), аналитическое выражение представленной ВАХ будет иметь вид

$$
\begin{aligned}
I(V)= & f\left(V_{2}\right)\left[\frac{k_{1} \exp (\alpha V)\left(I_{2}^{0}+k_{2} V\right)}{f\left(V_{1}\right) k_{1} \exp (\alpha V)+\left[1-f\left(V_{1}\right)\right]\left(I_{2}^{0}+k_{2} V\right)}\right] \\
& +\left[1-f\left(V_{2}\right)\right] k_{3} V .
\end{aligned}
$$

Здесь

$$
\begin{aligned}
& f\left(V_{1}\right)=\left[1+\exp \left(\frac{V-V_{1}}{\sigma_{1}}\right)\right]^{-1}, \\
& f\left(V_{2}\right)=\left[1+\exp \left(\frac{V-V_{2}}{\sigma_{2}}\right)\right]^{-1} .
\end{aligned}
$$

Здесь параметры $\sigma_{1}$ и $\sigma_{2}$ могут быть равными.

Сплошные кривые 2 и $2^{\prime}$ на рис. 3 демонстрируют аппроксимацию экспериментальной $\mathrm{BAX}$ с помощью выражений (4) и (1) соответственно. Видно, что для напряжения $V<6.0$ В кривая 2 значительно отличается от экспериментальной зависимости, а кривая $2^{\prime}$ (на рисунке она опущена на 100 единиц вниз) с ростом напряжения недопустимо отличается от измеренной кривой 1. Так, если в первом случае ошибка аппроксимации не превышает 50\%, то во втором случае она достигает сотен процентов. Причина этого - близкие значения наклонов объединяемых функций $I_{2}(V)$ и $I_{3}(V)$ при напряжениях $V>V_{1}$. Поэтому значительное превалирование одного процесса над другим может быть достигнуто при напряжениях, больших, чем использованные в эксперименте. 
Применение функций участия процессов в выражениях (2) и (5) позволяет улучшить совпадение кривых с точностью до единиц процентов и менее, что достигается подбором значения параметра $\sigma$. Кривая 3 на рис. 3 , полученная при значении $\sigma=2 \mathrm{~B}$, хорошо совпадает с экспериментальной зависимостью (кривая 1) во всем диапазоне независимой переменной, за исключением области $V_{1}<V<V_{2}$, где значение ошибки аппроксимации достигает $15 \%$. Уменьшение параметра $\sigma$ до значения 0.1 В приводит к совпадению расчетной и экспериментальной кривых до единиц процентов (кривая 4).

Полученная таким способом аппроксимирующая функция (7) далее может быть использована для получения аналитических выражений, описывающих другие характеристики исследуемого объекта и его параметры: зависимости сигнала фотопроводимости от смещения, вычисления автокорреляционной функции и теплового шума исследуемого полупроводника [8].

\section{4. Применение методики в расчетных задачах}

Предложенная методика составления аналитического выражения для описания действия параллельных и последовательных процессов, объединяемых в единый процесс, может быть использована при теоретическом исследовании явлений в физических системах. Продемонстрируем это на конкретном примере.

При рассмотрении процессов излучательной рекомбинации в квантовых ямах (КЯ) светодиодной гетероструктуры [26], когда в силу координатной зависимости уровня инжекции и числа уровней размерного квантования в мини-зонах число электронов в $n$-КЯ отличается от числа дырок в $p$-КЯ того же слоя активного вещества, встает задача записи аналитического выражения для расчета числа рекомбинируемых электроннодырочных пар в КЯ. Принципиальным является различие в концентрациях рекомбинируемых носителей заряда противоположных знаков. В этом случае не применимы соотношения, используемые в теории люминесценции полупроводников для описания излучательной рекомбинации [27], так как они получены в предположении пропорциональности скорости рекомбинации произведению концентраций электронов $n$ и дырок $p$. Поскольку из физических соображений ясно, что число рекомбинируемых пар „электрон-дырка“ $N_{\text {rec }}$ равно минимальному числу участвующих носителей заряда, расчет требуемого параметра удобно провести с использованием выражения (5). Тогда

$$
N_{\text {rec }}=\frac{n(x) p(x)}{f\left(x, x_{0}\right) n+\left[1-f\left(x, x_{0}\right)\right] p},
$$

где $f\left(x, x_{0}\right)=\left[1+\exp \left(\frac{x-x_{0}}{\sigma}\right)\right]^{-1} ; x_{0}-$ координата точки в полупроводнике, в которой концентрации электронов и дырок равны; $\sigma$ - параметр, характеризующий размер переходной области функции $f\left(x, x_{0}\right)$. Зная число рекомбинирующих электронно-дырочных пар, можно рассчитать ток инжекции в рассматриваемые КЯ, интенсивность генерируемого ею излучения и другие параметры светодиодной гетероструктуры.

\section{5. Заключение}

Предложена методика составления аналитического выражения экспериментальной кривой, имеющей один или несколько изломов. В зависимости от последовательного или параллельного участия парциальных процессов в результирующем процессе при составлении его аналитического выражения используются операции вычисления суммы процессов или вычисления их среднего геометрического. Принципиальное повышение точности аналитического выражения, описывающего экспериментальную кривую, достигается использованием функций участия процессов, которые зависят от координаты точки излома и параметра точности аппроксимации. Параметр точности определяет точность аппроксимации и подбирается из условия совпадения экспериментальной и аппроксимационной кривых.

Применение и возможности методики аппроксимации продемонстрированы на составлении аналитического выражения дифференциальной вольт-амперной характеристики фоторезистора из $n$-CdSe. Установлено, что точность аппроксимации может достигать единиц процентов, а полученное выражение стать основой для вычисления других свойств исследуемого объекта.

Показано, что предложенная методика может быть полезной при проведении аналитических расчетов, в которых необходимы выражения для вычисления комбинаций нескольких процессов, объединяемых в единый процесс по известному алгоритму.

\section{Список литературы}

[1] С.В. Булярский, Л.Н. Вострецова, С.А. Гаврилов. ФТП, 50 (1), 106 (2016).

[2] А.И. Михайлов. ФТП, 50 (1), 103 (2016).

[3] А.А. Ващенко, Д.О. Горячий, А.Г. Витухновский, Н.Н. Тананаев, В.А. Васнев, Е.Н. Родловская. ФТП, 50 (1), 120 (2016).

[4] Н.С. Курочкин, А.А. Ващенко, А.Г. Витухновский, П.Н. Тананаев. ФТП, 49 (7), 975 (2015).

[5] Г.В. Климко, Т.А. Комиссарова, С.В. Сорокин, Е.В. Контрош, Н.М. Лебедева, А.А. Усикова, Н.Д. Ильинская, В.С. Калиновский, С.В. Иванов. Письма ЖТФ, 41 (18), 82 (2015).

[6] Е.Г. Глуховский, Н.Д. Жуков. Письма ЖТФ, 41 (14), 47 (2016).

[7] Н.Е. Корсунская, Е.П. Шульга, Т.Р. Стара, П.М. Литвин, В.А. Бондаренко. ФТП, 50 (1), 112 (2016).

[8] В.Н. Давыдов. Электрон. техн. сер. 3, Микроэлектроника. 2, 43 (1982).

[9] В.Н. Давыдов, В.С. Гребенников, И.М. Мусина. Изв. вузов. Физика, 2, 90 (2012). 
[10] Ф.С. Габибов, Е.М. Зобов, М.Е. Зобов, С.П. Крамынин, Е.Г. Пашук, Ш.А. Халилов. Письма ЖТФ, 41 (8), 9 (2015).

[11] П.С. Вергелис, Е.Б. Якимов. ФТП, 49 (2), 149 (2015).

[12] Е.А. Гребенщикова, В.В. Евстропов, Н.Д. Ильинская, Ю.С. Мельников, О.Ю. Серебрякова, В.Г. Сидоров, В.В. Шерстнев, Ю.П. Яковлев. ФТП, 49 (3), 376 (2015).

[13] А.Ш. Абдинов, Р.Ф. Бабаева, Р.М. Рзаев, Н.А. Рагимова, С.И. Амирова. 50 (1), 35 (2016).

[14] Ш.Ш. Азимов, В.Н. Петухов, А.Н. Лакаев, А.М. Лексовский. ЖТФ, 86 (4), 155 (2016).

[15] А.В. Войцеховский, В.Н. Давыдов. Фотоэлектрические МДП-структуры из узкозонных полупроводников (Томск, Радио и связь, 1990).

[16] А.Н. Голубинский. Вестн. Воронеж. ин-та МВД России, 2, 24 (2007). Режим доступа:

http://cyberleninka.ru/article/n/metody-approksimatsii-eksperimentalnyh-dannyh-i-postroeniya-modeley

[17] Л.Д. Лямец. Мат. морфология: Электрон. мат. и медикобиол. журн. 1 (1), 12 (1996). Режим доступа: http://www.smolensk.ru/user/sgma/MMORPH/N-1-html/5.htm

[18] В.В. Малов, А.Р. Тамеев, С.В. Новиков, М.В. Хенкин, А.Г. Казанский, А.В. Ванников. ФТП, 50 (4), 489 (2016).

[19] Л.А. Косяченко, В.Я. Литвиненко, Е.Л. Маслянчук. ФТП, 50 (4), 515 (2016).

[20] В.Д. Попов. ФТП, 50 (3), 354 (2016).

[21] А.А. Зарубанов, К.С. Журавлев. ФТП, 49 (3), 392 (2016).

[22] Н.Д. Ильинский, С.А. Карандашев, Н.Г. Карпухина, Б.А. Матвеев, М.А. Ременный, Н.М. Стусь, А.А. Усикова. ФТП, 50 (5), 657 (2016).

[23] А.Н. Акимов, А.Э. Климов, И.Г. Неизвестный, В.Н. Шумский, В.С. Эпов. ФТП, 50 (4), 447 (2016).

[24] А.П. Одринский. ФТП, 49 (3), 294 (2015).

[25] В.Н. Давыдов, Д.А. Новиков. Докл. тУСУР, 1 (35), 64 (2015).

[26] Ф.Е. Шуберт. Светодиоды (М., Физматлит, 2008). [Пер. с англ. F.E. Schubert. Light-Emitted Diodes (Cambridge, 2006)].

[27] С. Зи. Физика полупроводниковых приборов. В 2-х кн. Кн. 1 (М., Мир, 1984).

Редактор Г.А. Оганесян

\section{Preparation of analytical expression of the physical process in experimental curve with a kinks}

\author{
V.N. Davydov, S.V. Kharitonov, N.E. Lugina, K.P. Melnik \\ Tomsk State University of Control Systems \\ and Radioelectronics, \\ 634050 Tomsk, Russia
}

Abstract The methodic of preparation of the analytical expression of the experimental curve having one or several kinks is proposed. Depending on the scheme of partial participation processes in the preparation of the resulting process a calculations of the sum of processes or their geometric average of value are used. For increase in precision of approximation ,participation functions" of processes which course is defined by the coordinate of a cross point of processes and „accuracy parameter" are injected into these expressions. Accuracy of approximation is established by their selection the corresponding measurement accuracy of the experimental curve. Application of the offered methodic in analytical calculations with a combination of several processes is shown, and also by preparation of the analytical expression of the experimental curve with kinks. Obtained in this way approximating expression it is characterized by obviousness, high precision of approximation, physical simplicity and may be used to calculate other properties of the semiconductor device. 